\title{
BLANCO-80: Thematic Collection of Structural and Thermodynamic Studies on Membranes
}

\author{
Alexey S. Ladokhin ${ }^{1} \cdot$ Ana-Nicoleta Bondar ${ }^{2}$ \\ Published online: 3 June 2020 \\ (c) Springer Science+Business Media, LLC, part of Springer Nature 2020
}

\section{Dear Colleagues,}

On behalf of the entire Editorial Team of the Journal of Membrane Biology, we are happy to announce the launching of a new Thematic Collection, BLANCO-80, committed to highlighting the advances and challenges in structural and thermodynamic characterization of membrane proteins and lipid bilayers. The collection is in honor of the occasion of the 80th birthday of Stephen H. White, who had made numerous seminal contributions to the field of Membrane Biophysics.

The first entry in the collection, entitled How we came to understand the "Tumultuous Chemical Heterogeneity" of the Lipid Bilayer Membrane, by William C. Wimley is published in the current issue. This insightful mini-review describes the fundamental principles behind the complete structure of the fluid lipid bilayer, deciphered by Stephen $\mathrm{H}$. White and his co-workers in the early 1990s, as well as the lasting implications of their pivotal work.

Encouraging and pioneering collaborations among experimentalists and theoreticians remains one of the many important contributions of Stephen H. White to the cultural landscape of lipid bilayer and membrane protein studies. The results of one such collaboration, that arose in part from the fertile ground of his laboratory, are presented in this issue.

Namely, the Experimental and Computational Characterization of Oxidized and Reduced Protegrin Pores in Lipid Bilayers by Rodnin and co-authors reports on the application of the methods of molecular dynamics simulations and fluorescence spectroscopy to gain structural and thermodynamic insights into lipid interactions of antimicrobial peptide Protegrin-1.

We invite you to contribute to this collection by submitting a Research Manuscript, Topical Review, Letter to the Editor or a Brief Comment to the Journal of Membrane Biology. Please indicate your intension to be associated with the $B L A N C O-80$ thematic collection in your cover letter. We are looking forward to your submissions.

Alexey S. Ladokhin, Editor-in-Chief

Ana-Nicoleta Bondar, Section Head Editor in Biophysics

Publisher's Note Springer Nature remains neutral with regard to jurisdictional claims in published maps and institutional affiliations.

This article is part of the Topical Collection: BLANCO-80:

Thematic Collection of Structural and Thermodynamic Studies on Membranes.

Alexey S. Ladokhin

aladokhin@kumc.edu

1 Department of Biochemistry and Molecular Biology, The University of Kansas Medical Center, Kansas City, KS 66160-7421, USA

2 Department of Physics, Theoretical Molecular Biophysics, Freie Universität Berlin, Arnimallee 14, 14195 Berlin, Germany 